\title{
Towards common European audio description guidelines: Results of the Pear Tree Project
}

Iwona Mazur, Agnieszka Chmiel

\begin{abstract}
The article reports on the Pear Tree Project (PTP), conducted as part of the Media for All project whose original aim was to develop audio description (AD) guidelines in Europe in order to ensure consistent high quality AD. However, before streamlining AD standards, a number of issues had to be addressed, the most essential one being whether relevant cross-linguistic and cross-cultural differences in Europe are insignificant enough to enable the development of such common European AD guidelines.

In order to answer these questions a methodology proposed by Chafe (1980) concerning the way representatives of various cultures and languages perceive and describe moving images was adopted by a group of AD researchers in the PTP. Participants from various countries were asked to watch a short film and recount what they saw. The data were then subjected to comparative lexical, discourse and narrative analysis in order to uncover both similarities and differences in the processing of visual information by representatives of the languages and cultures concerned. The results and their analysis will be presented in the paper on the basis of which the authors will attempt to provide an answer to the question of whether creating common European audio description guidelines is feasible.
\end{abstract}

Keywords: audio description, media accessibility, European audio description guidelines, film perception across languages and cultures

\section{Introduction}

Audio description (AD) has been developing very unevenly across Europe. In some countries (such as the UK) AD has already come of age, whereas in others (such as Greece) it is still at the crawling stage. As a result, countries belonging to the former category have worked out national AD standards and practices (which, however, differ from country to country and so does the quality of ADs), whereas those in the latter group are lagging far behind with no formal principles in place to guide audio describers in their work.

Given the above considerations and in view of the European Commission's Audiovisual Media Services Directive of 11 December 2007, which explicitly mentions the right of persons with disabilities to enjoy accessible media services, including through audio description, AD practitioners and researchers across Europe have become aware of the growing need to develop and standardise European $\mathrm{AD}$ guidelines in order to ensure consistent high quality AD practice. However, before European AD standards and practices can be streamlined, a number of issues have to be addressed, the most essential one being whether cross-linguistic and cross-cultural differences in Europe are not significant enough to prevent such common European AD guidelines from being developed.

In order to answer these questions a methodology proposed by Chafe (1980) and concerning the way representatives of various cultures and languages perceive and describe moving images was adopted by a group of AD researchers across Europe in the so-called Pear Tree Project (PTP). In the paper the results as well as their analysis will be presented, on the basis of which the authors will attempt to provide an answer to the question of whether creating common European audio description guidelines is a feasible undertaking. 


\section{Project rationale and objectives}

The Pear Tree Project was part of a larger Digital Television for All (DTV4All) project coordinated by Brunel University and aimed at facilitating the provision of access services on digital television across the European Union (http://www.psp-dtv4all.org/). The PTP was included in the audio description component of the DTV4All and was coordinated by Pilar Orero of Universitat Autònoma de Barcelona. The objective of the PTP was to answer the question whether it is possible to develop common European AD guidelines. Such guidelines would by all means help improve and even out the quality of AD across Europe. What is more, if guidelines common for all European countries were in place, it would be possible to translate $\mathrm{AD}$ scripts drafted in one language into a range of other languages. As such a solution would most probably be cheaper and quicker than drafting an AD script from scratch, the volume of audio described materials across Europe could significantly increase (cf. Orero 2008).

However, before the task of streamlining European AD guidelines could be undertaken, it seemed reasonable to verify whether people living in different European countries, with different linguistic and cultural backgrounds, perceive and interpret visual stimuli in a similar manner. To this end, the researchers involved in the PTP decided to employ a methodology developed for testing reception of the same visual input across languages and cultures, which methodology is described below.

\section{Methodology and materials}

The methodology used in the PTP was first developed by Professor Wallace Chafe of the University of California in Berkeley. In the mid-1970s, Chafe and his co-workers conducted a study called the Pear Stories Project (Chafe 1980), the primary objective of which was to find an interrelation between knowledge and manner of thought formulation. In particular the researchers wanted to find out how people talk about events in which they participated as well as how they describe them after some time.

The starting point for Chafe's experiment was a study conducted by Bartlett (1967), in which the subjects were asked to read a folktale and then write down what they remembered. Chafe, on the other hand, wanted the input to be provided in a non-verbal form and the subjects were to recount what they saw orally. Additionally, the study was to involve persons representing different languages and cultures in order to perform a cross-linguistic and crosscultural analysis.

The researchers, however, could not find a film that would meet their research criteria, which should include, among other things, the presentation of a series of events, some of which would be in sequence and some simultaneous. Some of the events were to be trivial, while other salient. Also, the visual input should provide for ambiguity of interpretation, but on the other hand should be interpretable by anyone. Due to the fact that none of the existing films met the above-mentioned criteria, the researchers decided to make a film especially for the purpose of the study, the so-called 'Pear Film'. The film was shot in California, in colour, with sound but with no dialogue. It lasted 6 minutes. It had simple editing, referred to universal experiences and did not include any culture-specific or historic references so that members of different cultures could understand it and recount it. The exact sequence of events was as follows (after Du Bois 1980: xii-xiii):

A man is on a ladder picking pears. He descends the ladder, kneels, and puts the pears from the pocket of his apron into one of three baskets below the pear tree. He removes a bandana from 
around his neck and wipes one of the pears. He then returns to the ladder and climbs back into the tree.

Toward the end of this sequence we hear the sound of a goat, and when the picker is back up the ladder, a man approaches with a goat on a leash. As they pass the baskets of pears, the goat strains towards them, but the man pulls him past and the two of them disappear into the distance.

We see another close up of the picker at his work, and then a boy approaches on a bicycle. He coasts in toward the baskets; stops, gets off his bike; looks up at the picker; puts down his bike; walks toward the baskets, again looking at the picker; picks up a pear; puts it back down; looks once more at the picker; and lifts up a basket full of pears. He puts the basket down near his bike; picks the bike up and gets on; picks up the basket and places it on the rack in front of the handlebars, and rides off. We again see the man continuing to pick pears.

The boy is now riding down the road, and we see a pear fall from the basket on his bike. Then we see a girl on a bicycle approaching from the other direction. As they pass, the boy turns to look at the girl, his hat flies off, and the front wheel of his bike hits a rock. The bike crashes, the basket falls off, and the pears spill onto the ground. The boy extricates himself from under the bike, and brushes off his leg.

In the meantime we hear what turns out to be the sound of a paddleball, and then we see three boys standing there, looking at the bike boy on the ground. The three pick up the scattered pears and put them back in the basket. The boy sets his bike upright, and two of the other boys lift the basket of pears back onto it. The bike boy begins walking his bike in the direction he was going, while the three other boys begin walking off in the other direction.

As they walk by the bike boy's hat on the road, the boy with the table-tennis bat toy sees it, picks it up, turns around, and we hear a loud whistle as he signals to the bike boy. The bike boy stops, takes three pears out of the basket, and holds them out as the other boy approaches with the hat. They exchange the pears and the hat, and the bike boy keeps going while the boy with the paddleball runs back to his two companions, to each of whom he hands a pear. They continue on eating their pears.

The scene now changes back to the tree, where we see the picker again descending the ladder. He looks at the two baskets, where earlier there were three, points at them, backs up against the ladder, shakes his head, and tips up his hat. The three boys are now seen approaching, eating their pears. The picker watches them pass by, and they walk off into the distance.

In the original study the subjects were shown the film and then were asked to tell what they saw. Their speeches were recorded and then transcribed. The subjects were asked to recount the film again after some time. The original experiment, conducted by Chafe in 1975, involved a group of English-language students at the University of California. Data for other languages was gathered at later stages, including Japanese (Clancy 1980, Downing 1980), German (Ehlers 1980), Greek (Tannen 1980), and Malaysian (Azia 1980), as referred to in Chafe (1980).

Given the fact that the above-mentioned experiment concerned both perception and description of visual input, the researchers in the DTV4ALL project assumed that its methodology could be applied in research on audio description with a view to answering the questions referred to in section 2 above.

\section{Data collection and participants}

The study was conducted in 11 countries and it involved 12 languages (including 10 European languages). Since the project concerns European AD guidelines, the data for non-European 
languages were discarded and only the data for the following European languages were analysed: Belgian Dutch (BE), Catalan (CAT), German (DE), Greek (EI), Spanish (ES), French (FR), Irish English (IE), Italian (IT), Polish (PL) and British English (UK) ${ }^{1}$. The reasons for selecting those particular languages were threefold. Firstly, we wanted to have a representation of the major European languages (such as English, French, German or Spanish). Secondly, we aimed at languages belonging to different language families (i.e. Germanic, Romance, Slavic or Greek). And finally, the third reason was a very practical one - our ultimate language selection depended on the willingness of audiovisual translation researchers in particular European countries to undertake the study and contribute to the project.

For each language included in the project there were approximately 20 subjects. In order to ensure statistical similarity, the participants in each country and across countries were a fairly homogenous group consisting in the vast majority of females, aged 17-25. They were all native speakers of the language in which they provided the descriptions. Most of the participants were university students in language departments, so the underlying assumption was that they all have good verbal and writing skills in the respective languages. Moreover, students of language departments at universities are usually females and this explains why the participants were mostly female. At this point, we assumed gender differences to be negligible for the purpose of this study.

\section{Procedure and data analysis}

A set of written instructions was developed and distributed among researchers conducting the study in their respective countries. According to the instructions, the participants were supposed to watch the Pear Film and write down what they saw. In the instructions there was no reference to the blind or audio description as such, so the assumption was that the subjects were to recount the film as if describing it to a person who simply had not seen it.

For the purpose of the experiment, the subjects were seated in a classroom, each in front of their own computer screen, with headphones on. They were allowed only one viewing of the film. As soon as the film was over, they were supposed to write their description of the events depicted in the film (in longhand, so that any corrections or deletions could be identified). There was no limitation as to the length of the descriptions. Similarly, the participants could take as long as they needed to complete their narratives. They were not supposed to talk to their peers while watching the film or when drafting the description. The researcher was constantly present in the room to supervise the entire procedure.

The subjects were also asked to fill in a biographical questionnaire providing information on age, sex, mother tongue, language used most often in their everyday communication and long stays abroad.

Once the experiment was carried out, the descriptions were transcribed on a computer (including deletions and corrections, if any), and then - for languages other than English they were translated into English in order to allow for a comparative analysis between the languages concerned. The researchers in the project were asked to analyse the data obtained for their respective languages according to an analysis template, which was based on data analysis from a study conducted by Tannen (1980) for American English and Greek. The analysis included the following 12 aspects: occurrence of the word 'film'; allusions to filmviewer perspective; verb tenses used in descriptions; giving pears to the boys; explanation of the cause of fall; man with the goat; taking pears; pearpicker's discovery; words used for the pearpicker; objects mentioned in the fall scene; terms for the threesome; reference to the toy. The idea behind all of these aspects as well as their potential significance for audio 
description research will be explained when discussing results obtained for each of the aspects (see section 6).

It should however once again be stressed that the primary aim of the study was to uncover the principles governing the perception of visual stimuli and the related narrative production by sighted viewers. This means that the project's findings should not be directly applied to $\mathrm{AD}$, as in the study the narrative production took place a posteriori and was targeted at sighted persons, whereas audio description is simultaneous with the described images and is directed at visually impaired persons. Nonetheless, the knowledge of how sighted viewers make sense of visual input could have implications for AD in that the effect created by AD on visually challenged viewers should be similar.

Also, and more importantly for the purpose of this study, if we establish that there are no significant differences in the way people across Europe perceive and talk about visual events, then we could make a tentative assumption (which would have to be tested in a separate study) that there would be no significant differences among persons with visual impairments belonging to the respective linguacultures in the way they perceive and respond to narratives. If this were the case, then we could assume that the creation of common European guidelines is possible.

\section{Results and discussion}

As the aim of the study was to discover potential analogies in perceiving and describing visual input across cultures, we were in general looking for similarities among the tested samples (i.e. results with no statistical significance) to be able to generalise and draw conclusions for all the languages concerned.

One of the statistical tests employed to this end was the Chi Square Test. It is used to measure the relationship between categorical variables (countries and aspects analysed in descriptions in the present case). As in any statistical tests, if the probability or $p$ value is less than 0.05 we may assume a statistically significant difference between the data, i.e. the tested populations really differ from one another. If the $p$ value exceeds 0.05 , there is no statistically significant difference and, in statistical terms, the populations do not differ and any difference observed is a coincidence. Therefore, the more results there are without statistical significance, the more similarities, and the more generalisations may be drawn as to the way visual input is described in countries and languages across Europe.

Table 1 below presents the results for Chi Square Tests conducted for all of the analysed aspects in the project.

Table 1. Chi Square Test results for all analysed aspects

\begin{tabular}{|l|l|}
\hline Aspect & Chi Square Test \\
\hline Occurrence of film & $\mathrm{p}<.05$ \\
\hline Allusions to film-viewer perspective & $\mathrm{p}<.001$ \\
\hline Verb tenses & $\mathrm{p}<.001$ \\
\hline Giving pears & $\mathrm{p}<.05$ \\
\hline Explanation of the cause of fall & $\mathrm{p}<.001$ \\
\hline Man with the goat & $\mathbf{p}=.77$ \\
\hline Taking pears & $\mathbf{p}<.001$ \\
\hline Pearpicker's discovery & $\mathbf{p}=. \mathbf{0 5 2}$ \\
\hline Words used for pearpicker & $\mathbf{p}<.001$ \\
\hline Objects mentioned in fall scene & $\mathbf{p}<.05$ \\
\hline Terms for the threesome & $\mathbf{p}<.05$ \\
\hline
\end{tabular}


\begin{tabular}{|l|l|}
\hline Reference to the toy & $\mathrm{p}<.001$
\end{tabular}

The obtained $p$ values show that in only 2 out of the 12 analysed aspects (i.e. 'man with goat' and 'pearpicker's discovery') the differences across the languages were statistically insignificant, which means that certain generalisations as regards narratives (and thus audio descriptions) in the respective languages can be made for those two aspects only. In other aspects of the analysis, the results differ too much to allow for generalisations. The results are discussed in detail below.

\subsection{Occurrence of the word 'film'}

In her analysis of American and Greek narratives Tannen (1980: 54) notes that Americans tended to discuss the Pear Film as a film whereas Greeks simply recounted the events depicted in the film without mentioning that the events actually happened in a film. In audio description the prevailing tendency is not to directly refer to the genre being described, be it a film or theatre performance. It should be noted however that the findings in this aspect would not be directly applicable to audio description, as $\mathrm{AD}$ assumes an audience who know that what they are watching is a film or a theater performance.

The researchers in the PTP wanted simply to see whether the sighted subjects in the respective countries would actually use the word 'film' or 'movie' in their narratives and if so, then audio descriptions should perhaps follow suit. Table 2 below presents percentages for the relevant data. Please note that a distinction was made between no reference to the word 'film' and one or more references to such word in a given description.

Table 2. Occurrence of the word 'film' - data

\begin{tabular}{|c|c|c|c|c|c|c|c|c|c|c|c|}
\hline & TOTAL & BE & CAT & DE & ES & FR & GR & IR & IT & PL & UK \\
\hline 0 & $63.3 \%$ & $66.7 \%$ & $80.0 \%$ & $83.3 \%$ & $45.0 \%$ & $80.0 \%$ & $70.0 \%$ & $53.1 \%$ & $45.5 \%$ & $45.0 \%$ & $80.0 \%$ \\
\hline$<1$ & $36.7 \%$ & $33.3 \%$ & $20.0 \%$ & $16.7 \%$ & $55.0 \%$ & $20.0 \%$ & $30.0 \%$ & $46.9 \%$ & $46.9 \%$ & $55.0 \%$ & $20.0 \%$ \\
\hline
\end{tabular}

The data show that in the vast majority of languages (i.e. 7 out of 10) most of the descriptions did not include the word 'film' or 'movie' (with the highest results for Catalan $-80.0 \%$, German $-83.3 \%$, French $-80.0 \%$, and British English - 80.0\%). The three languages in which reference to the word 'film' was made at least once in most of the texts were Spanish, Italian and Polish. It turns out that the differences among countries are statistically significant $(\mathrm{p}<.05$ in the Chi Square Test). This means that no generalisations can be made concerning this aspect since there is little similarity among the tested samples.

\subsection{Allusions to film-viewer perspective}

This aspect is similar to the one described in section 6.1. Here the researchers looked at the use of film metalanguage, such as cinema-associated jargon like "the camera pans" and "protagonist", phrases like "it shows", as well as phrases that presuppose an audience, e.g. "then we saw", "you could see", "I noticed". According to Tannen (1980: 60), "[a]11 these references serve to remind the listener that what is being talked about is a film".

According to the prevailing AD guidelines, film metalanguage should generally be avoided. It must be stressed that the applicability of the findings to AD drafting is again limited, as the aim of the audio describer is not the same as of the viewers in the project. In 
$\mathrm{AD}$ the listener needs not be reminded that what is described is a film or a performance (cf. 6.1 above). Nonetheless, it is interesting to see whether and how the sighted viewers use cinema-associated jargon in verbal reconstruction of visually presented narratives. Such jargon could then perhaps be included in $\mathrm{AD}$, but first it would have to be ascertained that it helps the blind visualise a given scene (which is beyond the scope of this project).

In the PTP the researchers wanted to find out whether narratives produced in the different languages include allusions to the film-viewer perspective. The results are presented in Table 3 below.

Table 3. Allusions to film-viewer perspective

\begin{tabular}{|c|c|c|c|c|c|c|c|c|c|c|c|}
\hline & TOTAL & BE & CAT & DE & ES & FR & GR & IR & IT & PL & UK \\
\hline 0 & $40.4 \%$ & $20.8 \%$ & $55.0 \%$ & $33.3 \%$ & $45.0 \%$ & $40.0 \%$ & $20.0 \%$ & $50.0 \%$ & $36.4 \%$ & $15.0 \%$ & $85.0 \%$ \\
\hline$<1$ & $59.6 \%$ & $79.2 \%$ & $45.0 \%$ & $66.7 \%$ & $55.0 \%$ & $60.0 \%$ & $80.0 \%$ & $50.0 \%$ & $63.6 \%$ & $85.0 \%$ & $15.0 \%$ \\
\hline
\end{tabular}

Contrary to the data obtained for references to the word 'film', here the majority of the narratives $(59.6 \%)$ did make one or more references to the film-viewer perspective (with the highest percentages for Dutch - 79.2\%, German - 66.7\%, Greek - 80.0\% and Polish $85.0 \%)$. The Chi-Square Test result $(\mathrm{p}<.001)$ shows that the differences among countries are significant and thus no generalisations across countries are possible in this respect..

\subsection{Verb tenses}

In this aspect of data analysis the tenses used in each text were judged and classified as follows: present only, past only, a mixture of present and past, past to present (i.e. the text starts in the past and then switches to the present until the end). The aim was to specify which tenses are usually used in describing films in a given culture. Table 3 below presents percentages broken down by language. Some languages, such as Dutch and Polish, manifest a clear preference for present tense $(87.5 \%$ and $70.0 \%$, respectively) while other show a different result (e.g. German, French and Greek - in $75.0 \%$ of texts a mixture of tenses was used).

Table 4. Verb tenses - data

\begin{tabular}{|c|c|c|c|c|c|c|c|c|c|c|c|}
\hline & TOTAL & BE & CAT & DE & ES & FR & GR & IR & IT & PL & UK \\
\hline present & $46.6 \%$ & $87.5 \%$ & $33.3 \%$ & $25.0 \%$ & $60.0 \%$ & $25.0 \%$ & $25.0 \%$ & $51.9 \%$ & $36.4 \%$ & $70.0 \%$ & $35.0 \%$ \\
\hline past & $5.3 \%$ & $4.2 \%$ & $9.5 \%$ & $0.0 \%$ & $5.0 \%$ & $0.0 \%$ & $0.0 \%$ & $18.5 \%$ & $0.0 \%$ & $5.0 \%$ & $5.0 \%$ \\
\hline mixed & $43.2 \%$ & $8.3 \%$ & $57.1 \%$ & $75.0 \%$ & $25.0 \%$ & $75.0 \%$ & $75.0 \%$ & $7.4 \%$ & $59.1 \%$ & $25.0 \%$ & $55.0 \%$ \\
\hline $\begin{array}{c}\text { past to } \\
\text { present }\end{array}$ & $4.9 \%$ & $0.0 \%$ & $0.0 \%$ & $0.0 \%$ & $10.0 \%$ & $0.0 \%$ & $0.0 \%$ & $22.2 \%$ & $4.5 \%$ & $0.0 \%$ & $5.0 \%$ \\
\hline
\end{tabular}

The statistical analysis reveals that these differences are significant among languages $(p<.001$ in a Chi-Square Test). To analyse the trends in more detail, a correspondence analysis was performed. This tool is used in statistics to analyse multi-way tables (such as Table 4 above) and results in a correspondence map or plot that presents the interrelations between variables and categories. The closer the data points on the map, the more correlated they are. Figure 1 presents the correspondence plot for verb tenses. 
This is an Author's Accepted Manuscript of an article published in Perspectives: Studies in Translatology 20: 5-23, available at:

http://www.tandfonline.com/doi/ref/10.1080/0907676X.2011.632687

Figure 1. Verb tenses - correspondence plot

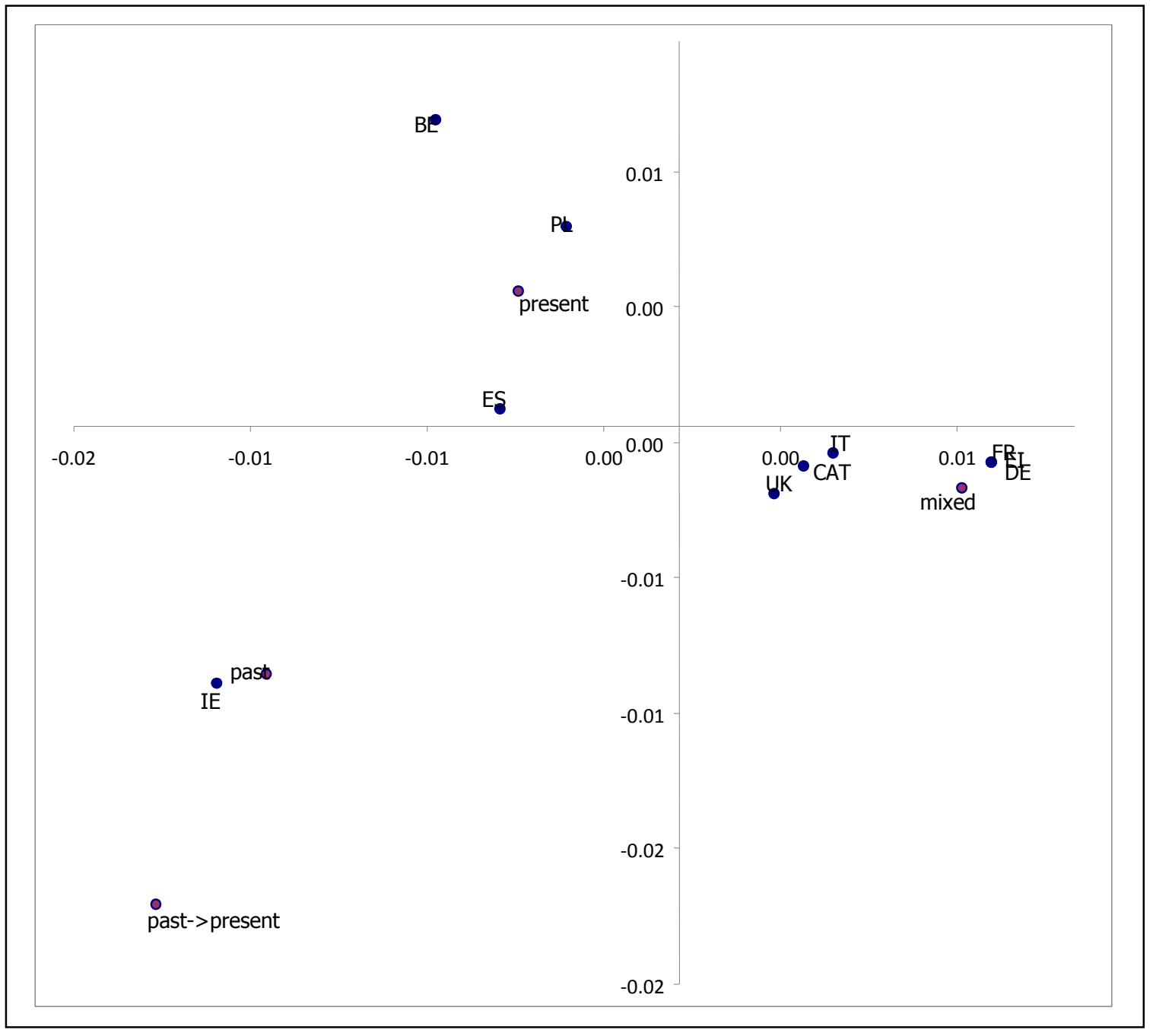

As can be seen from this graph, the variation is huge and points representing various languages are quite far apart. Greek, French, German, as well as Italian, British English and Catalan texts use a majority of mixed tenses (but note that the three first languages are closer to "mixed" so their preference is stronger than in the case of the latter three languages). Dutch and Irish are most separated from the rest of the languages so they vary the most in the present analysis.

\subsection{Giving pears to boys}

This aspect is related to the description of one event in the film, i.e. the boy giving pears to three boys. The results of the analysis provide information about: 
This is an Author's Accepted Manuscript of an article published in Perspectives: Studies in Translatology 20: 5-23, available at:

http://www.tandfonline.com/doi/ref/10.1080/0907676X.2011.632687

- how many texts do not refer to this event at all (no mention),

- how many texts only give a basic description, e.g. "the boy gave them a pear each" (basic),

- how many texts refer to this being a gesture of thanks, e.g. "the boy gave them a pear in return for their help" (thanks),

- how many texts make a moral judgment, e.g. "as he should do, the boy gave them a pear in return for their help" (moral judgment).

Such an aspect is analysed in the current study to see if and how the participants described and interpreted various events. Subjective interpretation of what is happening on screen is a bone of contention among AD scholars, with some fervently opposing any traces of subjective description and others accepting interpretation in certain contexts.

Table 5 below presents the overall data and percentages broken down by language. The majority of participants chose to interpret the event as a gesture of thanks $(59 \%)$. Variations in the results among languages are, again, quite large $(p<.05$ in the Chi Square Test). For instance, as many as $78.3 \%$ of Belgians interpreted this event as a gesture of thanks, while almost $38 \%$ of Poles did not mention this event at all.

Table 5. Giving pears to boys - data

\begin{tabular}{|c|c|c|c|c|c|c|c|c|c|c|c|}
\hline & TOTAL & BE & CAT & DE & ES & FR & GR & IR & IT & PL & UK \\
\hline basic & $19.6 \%$ & $8.7 \%$ & $20.0 \%$ & $27.3 \%$ & $25.0 \%$ & $0.0 \%$ & $25.0 \%$ & $21.7 \%$ & $38.1 \%$ & $20.0 \%$ & $11.8 \%$ \\
\hline $\begin{array}{c}\text { no } \\
\text { mention }\end{array}$ & $19.1 \%$ & $13.0 \%$ & $5.0 \%$ & $36.4 \%$ & $20.0 \%$ & $21.1 \%$ & $35.0 \%$ & $17.4 \%$ & $23.8 \%$ & $20.0 \%$ & $5.9 \%$ \\
\hline thanks & $59.3 \%$ & $78.3 \%$ & $70.0 \%$ & $36.4 \%$ & $45.0 \%$ & $78.9 \%$ & $40.0 \%$ & $56.5 \%$ & $38.1 \%$ & $60.0 \%$ & $82.3 \%$ \\
\hline $\begin{array}{c}\text { moral } \\
\text { jud. }\end{array}$ & $2.1 \%$ & $0.0 \%$ & $5.0 \%$ & $0.0 \%$ & $10.0 \%$ & $0.0 \%$ & $0.0 \%$ & $4.3 \%$ & $0.0 \%$ & $0.0 \%$ & $0.0 \%$ \\
\hline
\end{tabular}

The differences are clearer when depicted as a correspondence plot (Figure 4). In fact, the distances between points for various languages are large, with the exception of German and Greek or Dutch, British English and French. 
This is an Author's Accepted Manuscript of an article published in Perspectives: Studies in Translatology 20: 5-23, available at:

http://www.tandfonline.com/doi/ref/10.1080/0907676X.2011.632687

Figure 2. Giving pears to boys - correspondence plot

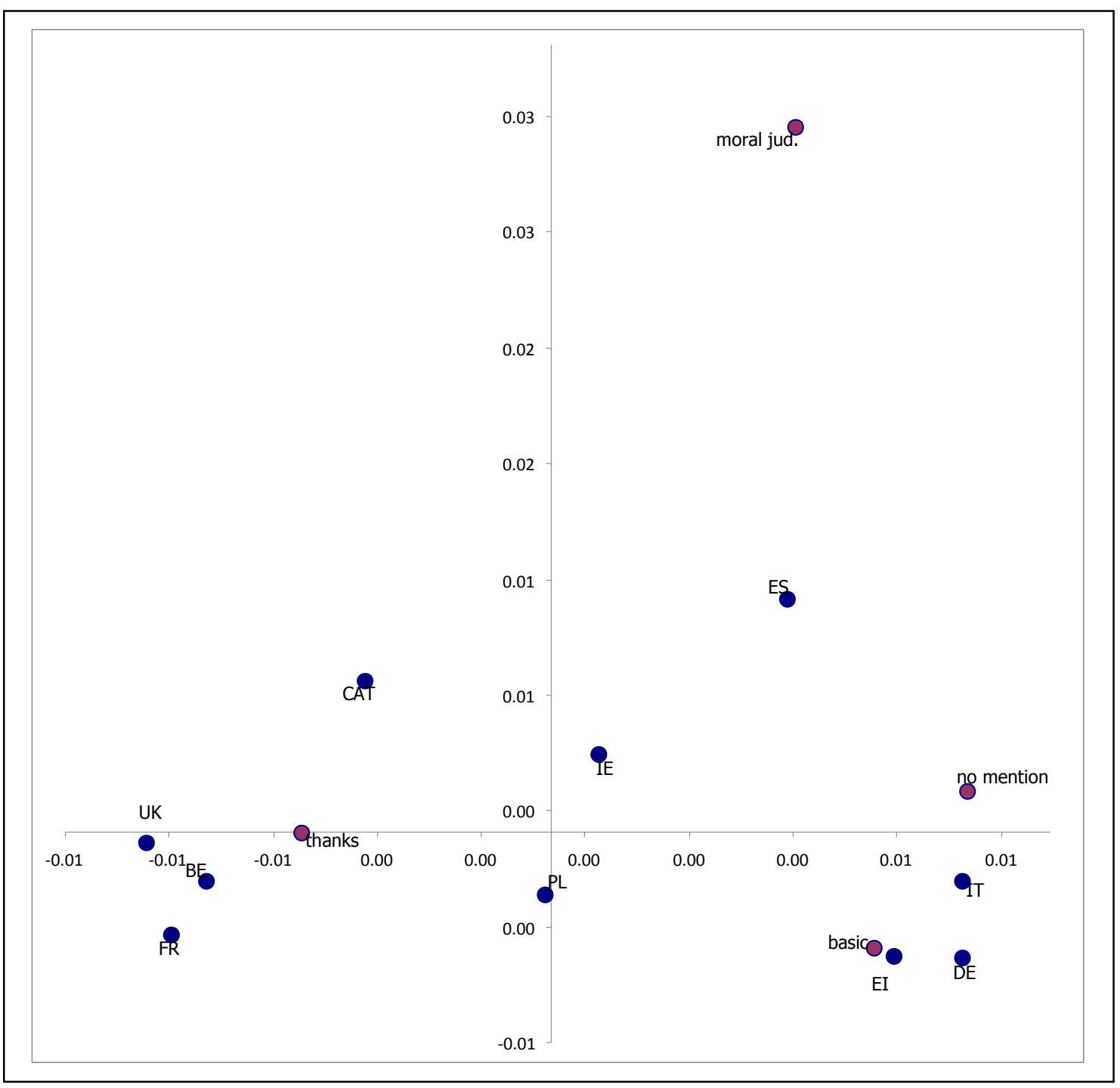

It is obvious that moral judgment was the least preferred solution, which (despite statistically significant differences among the data) can serve as a hint to audio describers.

\subsection{Explanations of the cause of fall}

This aspect is also related to the interpretation of events. The original Pear Film was recorded so that the cause of the boy's fall is ambiguous. As discovered by Tannen (1980: 73), "although causality is not clearly discernible in the film, most speakers (...) speculate about or impute causality in their stories". The same is true for our data, which manifests statistically significant differences among languages $(p<.001$ in the Chi Square Test). The respondents answers were classified, following Tannen (1980), as the following causes:

- turning and hitting rock, 
This is an Author's Accepted Manuscript of an article published in Perspectives: Studies in Translatology 20: 5-23, available at:

http://www.tandfonline.com/doi/ref/10.1080/0907676X.2011.632687

- tripping on rock,

- looking at girl,

- meeting with girl,

- collision with girl,

- rushing,

- tipping hat.

Table 6 presents the respondents' answers.

Table 6. Explanations of the cause of fall - data

\begin{tabular}{|c|c|c|c|c|c|c|c|c|c|c|c|}
\hline & TOTAL & BE & CAT & DE & ES & FR & GR & IR & IT & PL & UK \\
\hline $\begin{array}{c}\text { turn and } \\
\text { rock }\end{array}$ & $38.7 \%$ & $61.9 \%$ & $35.7 \%$ & $66.6 \%$ & $16.7 \%$ & $31.6 \%$ & $0.0 \%$ & $47.4 \%$ & $17.6 \%$ & $50.0 \%$ & $63.1 \%$ \\
\hline $\begin{array}{c}\text { tripping on } \\
\text { rock }\end{array}$ & $26.0 \%$ & $33.3 \%$ & $35.7 \%$ & $11.1 \%$ & $33.3 \%$ & $31.6 \%$ & $0.0 \%$ & $26.3 \%$ & $29.4 \%$ & $30.0 \%$ & $21.0 \%$ \\
\hline $\begin{array}{c}\text { looking at } \\
\text { girl }\end{array}$ & $12.7 \%$ & $4.8 \%$ & $7.1 \%$ & $11.1 \%$ & $11.1 \%$ & $5.3 \%$ & $41.2 \%$ & $0.0 \%$ & $23.5 \%$ & $15.0 \%$ & $10.5 \%$ \\
\hline $\begin{array}{c}\text { meeting } \\
\text { with girl }\end{array}$ & $5.2 \%$ & $0.0 \%$ & $0.0 \%$ & $0.0 \%$ & $22.2 \%$ & $0.0 \%$ & $0.0 \%$ & $21.0 \%$ & $5.9 \%$ & $0.0 \%$ & $0.0 \%$ \\
\hline collision & $5.2 \%$ & $0.0 \%$ & $7.1 \%$ & $0.0 \%$ & $11.1 \%$ & $10.5 \%$ & $0.0 \%$ & $5.3 \%$ & $5.9 \%$ & $5.0 \%$ & $5.3 \%$ \\
\hline rushing & $2.3 \%$ & $0.0 \%$ & $0.0 \%$ & $0.0 \%$ & $0.0 \%$ & $0.0 \%$ & $17.6 \%$ & $0.0 \%$ & $5.9 \%$ & $0.0 \%$ & $0.0 \%$ \\
\hline hat & $9.8 \%$ & $0.0 \%$ & $14.3 \%$ & $11.1 \%$ & $5.6 \%$ & $21.1 \%$ & $41.2 \%$ & $0.0 \%$ & $11.8 \%$ & $0.0 \%$ & $0.0 \%$ \\
\hline
\end{tabular}

In total, the descriptions favoured the first cause (turning and hitting rock $-38.7 \%$ ). Some languages manifested a preference for very few possible causes. For instance, only three causes were identified in Greek texts (looking at girl $-41.2 \%$; rushing $-17.6 \%$ and tipping hat $-41.2 \%$ ).

Figure 3. Explanations of the cause of fall - correspondence plot

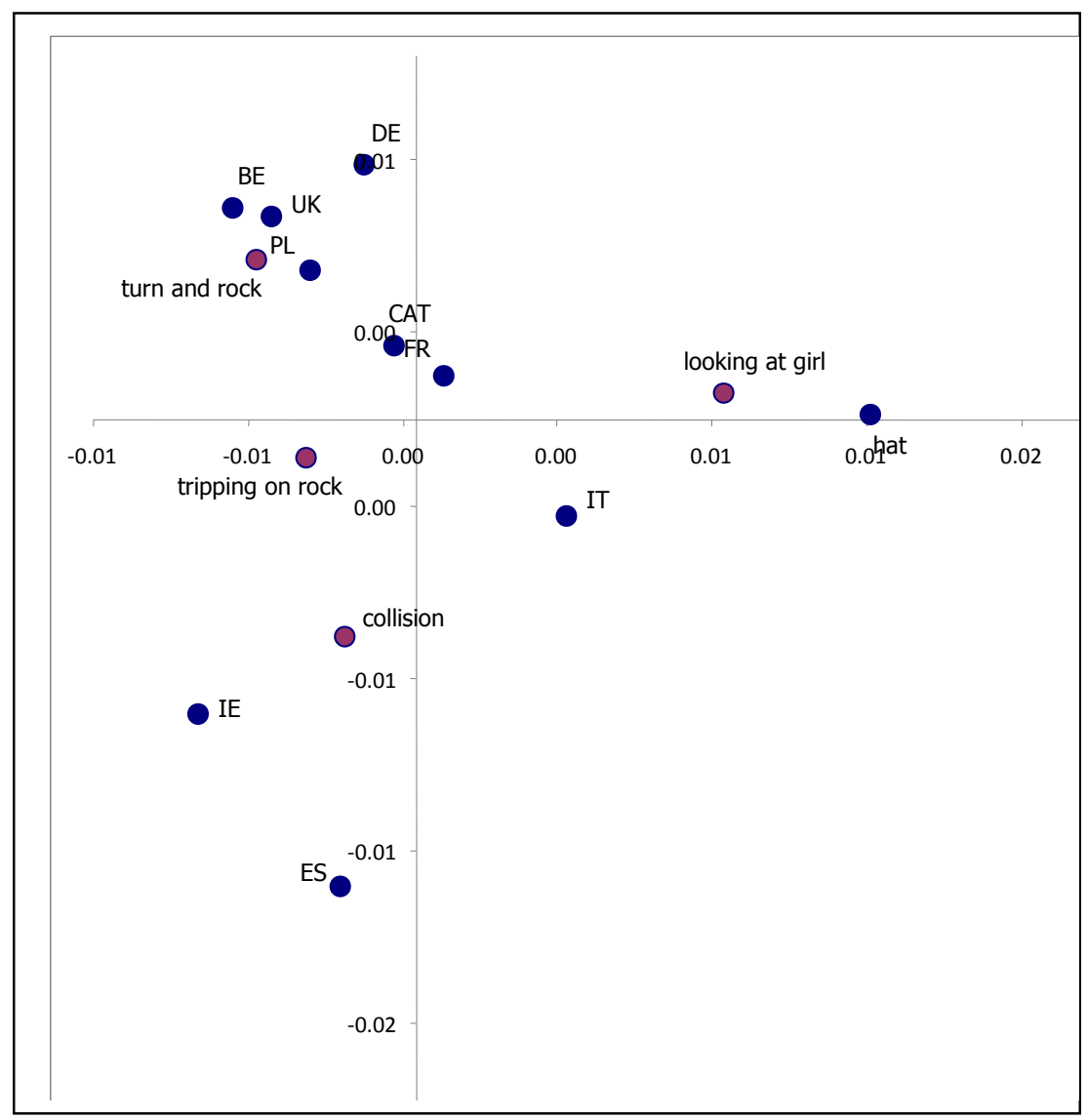


The correspondence plot shows some trends, such as a cluster of languages (British English, Polish, Dutch, German) near the cause "turning and hitting rock", suggesting a similarity for these languages. Italian, Greek, Spanish and Irish data points are more separated and differ the most from the rest. However, due to the huge variation in results and many possible causes, it is difficult to draw any generalisations from the analysis of this aspect of the descriptions.

\subsection{Man with the goat}

At the very beginning, the film depicts a man leading a goat. The man walks past the pear tree but has no influence on the film narrative. The analysis of this aspect is relevant for audio description because audio describers frequently have to choose what to describe and what to omit and they also have to decide whether events directly unrelated to the sequence of events in a film should be reflected in audio description. The episode with the man and the goat is irrelevant to the story described in the experiment. It is thus interesting to see if it was omitted or included in the descriptions. The Chi Square Test shows no statistically significant difference among languages $(p=.77)$, which means that the countries present similar preferences and generalisations can be made based on the obtained data.

Table 7. Man with the goat - data

\begin{tabular}{|c|c|c|c|c|c|c|c|c|c|c|c|}
\hline & TOTAL & BE & CAT & DE & ES & FR & GR & IR & IT & PL & UK \\
\hline mention & $42.5 \%$ & $50.0 \%$ & $44.4 \%$ & $50.0 \%$ & $31.0 \%$ & $44.4 \%$ & $28.6 \%$ & $41.2 \%$ & $38.9 \%$ & $50.0 \%$ & $39.4 \%$ \\
\hline no mention & $57.5 \%$ & $50.0 \%$ & $55.6 \%$ & $50.0 \%$ & $69.0 \%$ & $55.6 \%$ & $71.4 \%$ & $58.8 \%$ & $61.1 \%$ & $50.0 \%$ & $60.6 \%$ \\
\hline
\end{tabular}

As Table 7 above presents, the narrow majority (57.5\%) of texts does not mention the event with Greeks and Spaniards having a more pronounced preference. However, the difference is not convincing enough $(57.5 \%$ versus $42.5 \%)$ and we may conclude that although the studies manifest similar results across countries (no significant difference) we still cannot generalise because the respondents are equally indecisive. Thus, further studies are needed in this respect.

\subsection{Taking pears}

This aspect is similar to the one described in section 6.4, i.e. the study seeks to answer the question whether the descriptions mention the event of taking pears by the bicycle boy and if these descriptions are neutral (basic) or interpreted as theft (stealing) or even judged from a moral standpoint (moral judgment). The general results presented in Table 8 show that most texts include either a basic description $(50.6 \%)$ or an interpretation of the event as an act of stealing (45.4\%). However, the differences between languages are statistically significant $(p<.001$ in the Chi Square Test) and a closer scrutiny reveals a lot of variation in the data. For instance, the majority of Catalans interpret the event (75.0\%) while Germans are much more objective and present a basic description in $83.3 \%$ of their texts (similarly to Poles $-90 \%$ of basic descriptions). Spaniards, the Irish and Italians are the only groups that manifested any moral judgement in this context (with $5.0 \%, 5.0 \%$ and $4.0 \%$ of texts with moral judgment of the event respectively). 
This is an Author's Accepted Manuscript of an article published in Perspectives: Studies in Translatology 20: 5-23, available at:

http://www.tandfonline.com/doi/ref/10.1080/0907676X.2011.632687

Table 8. Taking pears - data

\begin{tabular}{|c|c|c|c|c|c|c|c|c|c|c|}
\hline & TOTAL & CAT & DE & ES & FR & GR & IR & IT & PL & UK \\
\hline no mention & $2.3 \%$ & $0.0 \%$ & $0.0 \%$ & $5.0 \%$ & $0.0 \%$ & $15.0 \%$ & $0.0 \%$ & $0.0 \%$ & $0.0 \%$ & $0.0 \%$ \\
\hline basic & $50.60 \%$ & $25.0 \%$ & $83.3 \%$ & $30.0 \%$ & $36.8 \%$ & $60.0 \%$ & $55.0 \%$ & $44.0 \%$ & $90.0 \%$ & $44.4 \%$ \\
\hline stealing & $45.4 \%$ & $75.0 \%$ & $16.7 \%$ & $60.0 \%$ & $63.2 \%$ & $25.0 \%$ & $40.0 \%$ & $52.0 \%$ & $10.0 \%$ & $55.5 \%$ \\
\hline moral jud. & $1.7 \%$ & $0.0 \%$ & $0.0 \%$ & $5.0 \%$ & $0.0 \%$ & $0.0 \%$ & $5.0 \%$ & $4.0 \%$ & $0.0 \%$ & $0.0 \%$ \\
\hline
\end{tabular}

The correspondence plot in Figure 4 shows that Greek texts differ the most from the rest, while French, Spanish, Italian, British English and Catalan texts are more similar with their preference for the interpretation of the event as stealing.

Figure 4. Taking pears - correspondence plot

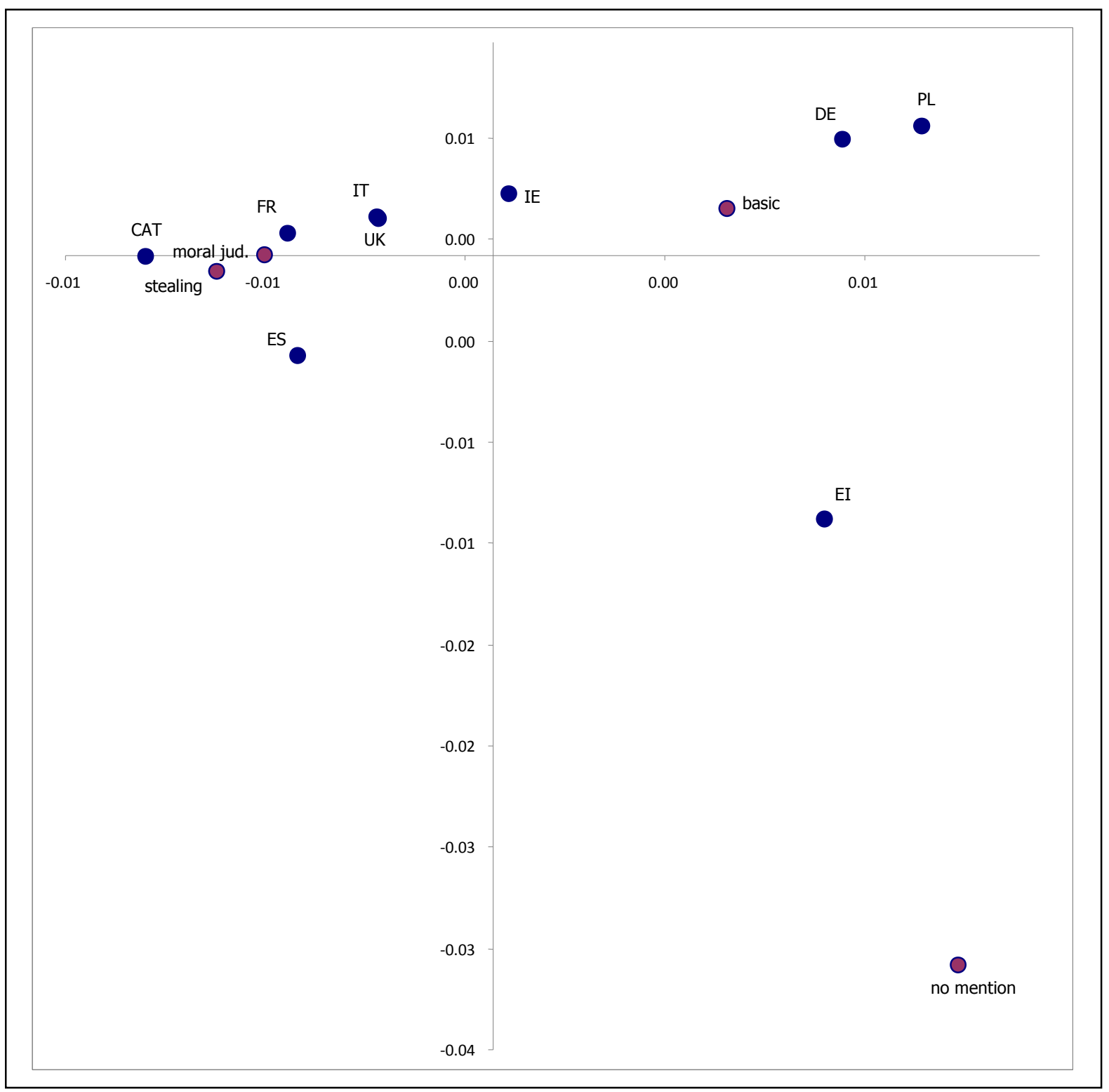

\subsection{Pearpicker's discovery}

This aspect is similar to the previous one and pertains to the interpretation of events and emotions. The description of emotions, just like interpretation of events, is a controversial 
issue in audio description. The proponents of objectivity claim that blind audiences have the right to determine what emotions the character manifests by themselves. The opponents underline that the human face and mimicry is so complex and ambiguous that it is sometimes very difficult to verbalise a facial expression without explicitly naming it.

In the last scene of the film the pearpicker notices that one basket of pears is missing and immediately afterwards he sees three boys walking by and eating pears. The descriptions in the texts were classified as no mention, basic (when an objective description was given), describing emotions or reaction, interpretive or describing both the pearpicker's emotions and reaction.

The Chi Square Test $(p=.52)$ shows no statistically significant differences among languages which justifies generalisations across all groups of respondents. However, as in the case of the sixth aspect, the results are not conclusive. On average, $35.3 \%$ of texts give basic descriptions, while interpretations are presented in $14.4 \%$ of texts.

Table 9. Pearpicker's discovery - data

\begin{tabular}{|c|c|c|c|c|c|c|c|c|c|c|}
\hline & TOTAL & CAT & DE & ES & FR & GR & IR & IT & PL & UK \\
\hline no mention & $10.8 \%$ & $5.0 \%$ & $10.0 \%$ & $10.0 \%$ & $5.3 \%$ & $20.0 \%$ & $15.0 \%$ & $22.2 \%$ & $0.0 \%$ & $10.0 \%$ \\
\hline basic & $35.3 \%$ & $20.0 \%$ & $40.0 \%$ & $35.0 \%$ & $36.8 \%$ & $25.0 \%$ & $50.0 \%$ & $50.0 \%$ & $35.0 \%$ & $30.0 \%$ \\
\hline $\begin{array}{c}\text { describe } \\
\text { emotions or } \\
\text { reaction }\end{array}$ & $30.5 \%$ & $40.0 \%$ & $30.0 \%$ & $40.0 \%$ & $42.1 \%$ & $10.0 \%$ & $25.0 \%$ & $11.1 \%$ & $45.0 \%$ & $30.0 \%$ \\
\hline interpret & $14.4 \%$ & $5.0 \%$ & $10.0 \%$ & $15.0 \%$ & $5.3 \%$ & $35.0 \%$ & $10.0 \%$ & $5.6 \%$ & $15.0 \%$ & $25.0 \%$ \\
\hline $\begin{array}{c}\text { emotions and } \\
\text { reaction }\end{array}$ & $9.0 \%$ & $30.0 \%$ & $10.0 \%$ & $0.0 \%$ & $10.5 \%$ & $10.0 \%$ & $0.0 \%$ & $11.1 \%$ & $5.0 \%$ & $5.0 \%$ \\
\hline
\end{tabular}

Table 9 depicts detailed data broken down by languages. Interpretations are most frequent in Greek texts (35.0\%) and rarest in Catalan texts (5.0\%). We may conclude that countries are equally indecisive when it comes to the description of such an ambiguous event. Once again, more studies are needed in this area.

\subsection{Words used for pearpicker}

This aspect of the analysis focused on the words used to denote the pearpicker. As Tannen (1980: 69) claimed: "narratives exhibit interpretation not only in their explicit statements about the actions and characters" but also through lexical choice. Like Tannen, we applied the following classification: non-interpretive (e.g. man, guy), particular (e.g. pearpicker, protagonist) and interpretive (e.g. farmer, Chicano man).

Table 10. Words used for pearpicker - data

\begin{tabular}{|c|c|c|c|c|c|c|c|c|c|c|c|}
\hline & TOTAL & BE & CAT & DE & ES & FR & GR & IR & IT & PL & UK \\
\hline $\begin{array}{c}\text { non- } \\
\text { interpretive }\end{array}$ & $51.8 \%$ & $50.0 \%$ & $46.2 \%$ & $76.9 \%$ & $30.5 \%$ & $56.7 \%$ & $45.0 \%$ & $72.2 \%$ & $46.3 \%$ & $60.5 \%$ & $53.1 \%$ \\
\hline particular & $18.8 \%$ & $37.5 \%$ & $0.0 \%$ & $23.1 \%$ & $0.0 \%$ & $43.3 \%$ & $0.0 \%$ & $22.2 \%$ & $7.3 \%$ & $34.2 \%$ & $31.3 \%$ \\
\hline interpretive & $29.4 \%$ & $12.5 \%$ & $53.8 \%$ & $0.0 \%$ & $69.5 \%$ & $0.0 \%$ & $55.0 \%$ & $5.6 \%$ & $46.3 \%$ & $5.3 \%$ & $15.6 \%$ \\
\hline
\end{tabular}

The differences among languages were, again, statistically significant $(p<.001$ in the Chi Square Test). In total, $51.8 \%$ of texts used non-interpretive words to denote a pearpicker, $18.8 \%$ used particular words and $29.4 \%$ used interpretive words. 
This is an Author's Accepted Manuscript of an article published in Perspectives: Studies in Translatology 20: 5-23, available at:

http://www.tandfonline.com/doi/ref/10.1080/0907676X.2011.632687

Figure 5. Words used for pearpicker - correspondence plot

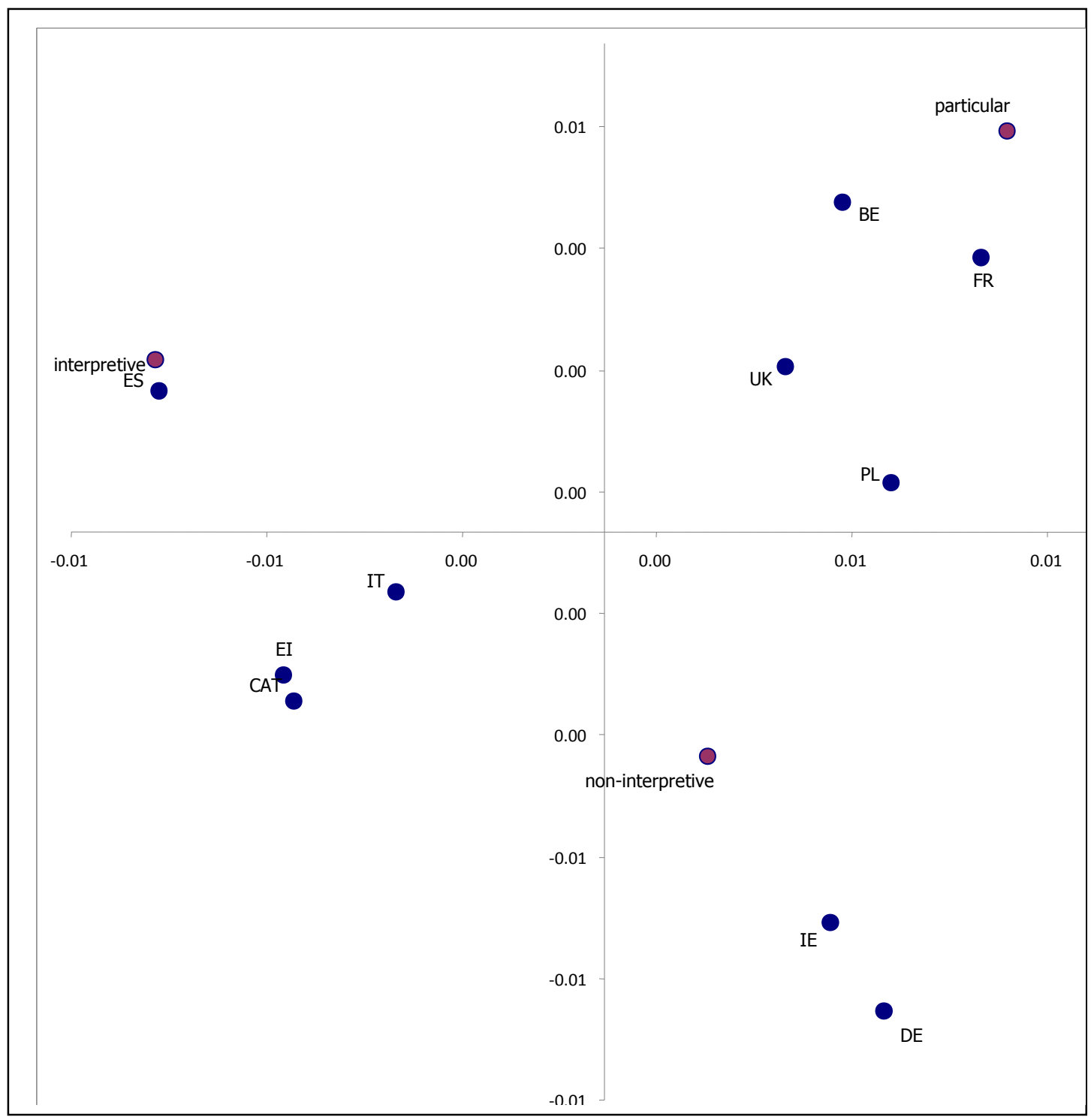

The correspondence plot in Figure 5 shows that Spaniards chose interpretive descriptions most frequently while Polish, British, Belgian and French respondents showed a slightly similar preference for particular words.

\subsection{Objects mentioned in fall scene}

This aspect focuses on the objects mentioned in the boy's fall from a bike. The cause of the event is ambiguous, although most respondents attributed the fall to the boy's turning and tripping on rock (see section 6.5). It is interesting to see if the descriptions include additional 
This is an Author's Accepted Manuscript of an article published in Perspectives: Studies in Translatology 20: 5-23, available at:

http://www.tandfonline.com/doi/ref/10.1080/0907676X.2011.632687

elements (even if they are not interpreted as having caused the fall) simply because they were visible on the screen. The differences among countries are significant $(p<.05$ in the Chi Square Test) so no results can be generalised across all the tested samples. Poles $(85.0 \%)$ and Belgians (75.0\%) are the only respondents to have included all elements (girl, rock, hat) in their descriptions.

Table 11. Objects mentioned in fall scene - data

\begin{tabular}{|c|c|c|c|c|c|c|c|c|c|c|c|}
\hline & TOTAL & $\mathrm{BE}$ & CAT & $\mathrm{DE}$ & ES & FR & GR & IR & IT & $\mathrm{PL}$ & UK \\
\hline fall & $5.6 \%$ & $0.0 \%$ & $5.3 \%$ & $0.0 \%$ & $15.8 \%$ & $0.0 \%$ & $11.8 \%$ & $7.7 \%$ & $10.0 \%$ & $0.0 \%$ & $5.0 \%$ \\
\hline girl & $7.1 \%$ & $0.0 \%$ & $5.3 \%$ & $0.0 \%$ & $10.5 \%$ & $15.0 \%$ & $0.0 \%$ & $11.5 \%$ & $10.0 \%$ & $0.0 \%$ & $15.0 \%$ \\
\hline rock & $3.0 \%$ & $0.0 \%$ & $5.3 \%$ & $0.0 \%$ & $5.3 \%$ & $0.0 \%$ & $0.0 \%$ & $7.7 \%$ & $10.0 \%$ & $0.0 \%$ & $0.0 \%$ \\
\hline hat & $0.5 \%$ & $0.0 \%$ & $0.0 \%$ & $0.0 \%$ & $5.3 \%$ & $0.0 \%$ & $0.0 \%$ & $0.0 \%$ & $0.0 \%$ & $0.0 \%$ & $0.0 \%$ \\
\hline girl and hat & $12.7 \%$ & $16.7 \%$ & $15.8 \%$ & $0.0 \%$ & $15.8 \%$ & $25.0 \%$ & $17.6 \%$ & $11.5 \%$ & $15.0 \%$ & $5.0 \%$ & $0.0 \%$ \\
\hline hat and rock & $1.0 \%$ & $0.0 \%$ & $0.0 \%$ & $8.3 \%$ & $0.0 \%$ & $0.0 \%$ & $0.0 \%$ & $0.0 \%$ & $0.0 \%$ & $0.0 \%$ & $5.0 \%$ \\
\hline girl and rock & $19.3 \%$ & $8.3 \%$ & $10.5 \%$ & $25.0 \%$ & $15.8 \%$ & $0.0 \%$ & $29.4 \%$ & $23.1 \%$ & $25.0 \%$ & $10.0 \%$ & $50.0 \%$ \\
\hline girl, rock, hat & $50.8 \%$ & $75.0 \%$ & $57.9 \%$ & $66.6 \%$ & $31.6 \%$ & $60.0 \%$ & $41.2 \%$ & $38.5 \%$ & $30.0 \%$ & $85.0 \%$ & $25.0 \%$ \\
\hline
\end{tabular}

Figure 6. Objects mentioned in fall scene - correspondence plot

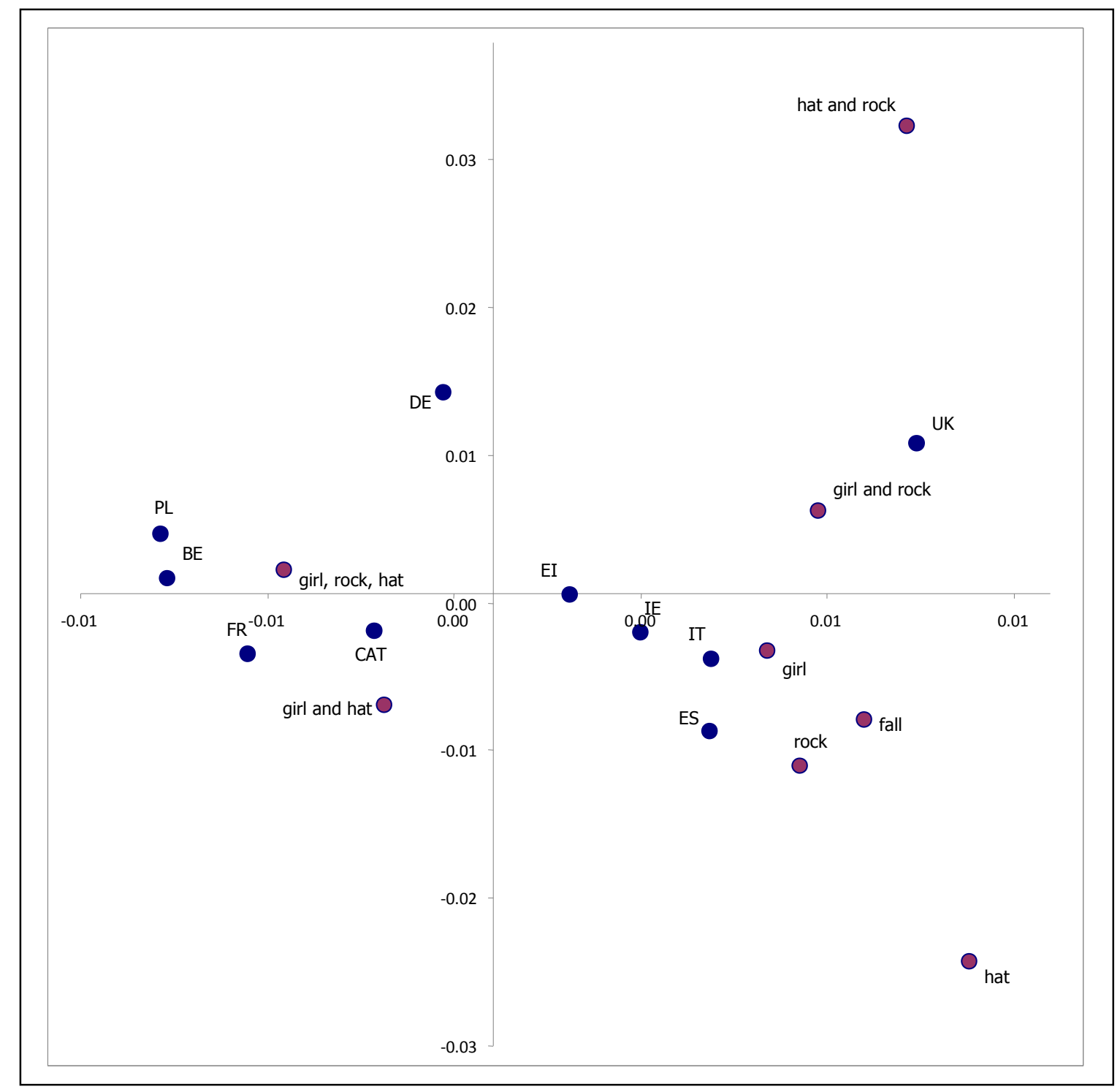


The correspondence plot in Figure 6 proves a great variation in the data across languages so, once again, no sufficiently justified generalisations are possible.

\subsection{Terms for the threesome}

This aspect focuses on stylistic variation. The more terms for the three boys are used in the text, the more stylistically varied they are in this respect. Descriptions in various languages differed $(\mathrm{p}<.05$ in the Chi Square Test) with some depicting huge variations (22 different terms in Italian texts, 18 in Dutch texts and 17 in German texts) and others using many repetitions (only 3 different terms used by Greeks) (Table 11).

Table 12. Terms for the threesome - data

\begin{tabular}{|c|c|c|c|c|c|c|c|c|c|c|c|}
\hline & TOTAL & BE & CAT & DE & ES & FR & GR & IR & IT & PL & UK \\
\hline no. of terms used & 114 & 18 & 10 & 17 & 8 & 7 & 3 & 10 & 22 & 11 & 8 \\
\hline
\end{tabular}

The differences could possibly be explained in terms of some languages having more synonyms for the concept of a 'threesome' than others. Another explanation could be that in some languages (such as Polish) repetition is considered bad style and it is recommended to use as many synonyms as possible, whereas in other languages (such as English) the use of repetitions is more acceptable. This therefore may offer some recommendations for audio describers in various countries as to whether to use synonyms and avoid repetitions.

\subsection{Reference to the toy}

The final aspect of the analysis was to show what to do in audio description with objects that have no specific names in a given language. The toy is a table-tennis bat with a ball attached to it with a string. It turns out that Spaniards, the French and Italians were more willing to omit this detail (possibly because of the lack of a term) in their descriptions than Poles, for instance. Belgians used references to the toy most often and this can be explained by the fact that Dutch is the only language from among the ones present in the study with a simple term denoting the said toy (Table 13).

Table 13. Reference to the toy - data

\begin{tabular}{|c|c|c|c|c|c|c|c|c|c|c|c|}
\hline & TOTAL & BE & CAT & DE & ES & FR & GR & IR & IT & PL & UK \\
\hline no. of references & 75 & 22 & 10 & 6 & 1 & 2 & 4 & 5 & 3 & 16 & 6 \\
\hline
\end{tabular}

\section{Conclusions (and some methodological considerations)}

The Pear Tree Project aimed at finding general characteristics of describing film narratives across 12 (including 10 European) languages in order to determine whether common European AD standards are possible. The analysis, which focused on European languages only, shows that we cannot really generalise about film descriptions in these languages because the analysed texts manifested huge variations. The general assumption was that if there are no statistically significant differences among languages, the results may be 
interpreted as depicting characteristics common for all of them. However, statistically significant differences were found in 10 out of 12 examined aspects. The results were similar enough to lead to generalizations in references to the man with a goat (aspect 6) and in descriptions about the discovery of the missing pears (aspect 8). Unfortunately, the results of the analysis in these two aspects were inconclusive. The difference between the number of texts mentioning the irrelevant man with a goat and not mentioning him was small, although the majority of texts applied the latter option. When it comes to the pearpicker's discovery $35.0 \%$ of texts included basic descriptions and $32.0 \%$ described the pearpicker's emotions or reaction. No solution was predominant and more research is required in this area to see if similar events are objectively described or interpreted.

The differences across languages in the ten remaining aspects are too huge to allow justified generalisations and, additionally, some opposing tendencies or trends may be observed. For instance, the majority of descriptions did not include the word 'film' or its synonyms but, on the other hand, the majority of texts included allusions to film-viewer perspective. The act of giving pears was interpreted as a gesture of gratitude in $57.0 \%$ of texts but the act of taking pears was not interpreted in $54.0 \%$ of cases.

In general, variations disclosed in the statistical analysis of correspondence are extensive across languages. Where the texts from specific countries do not differ, the results are equally inconclusive. It could be the case that this is due to some methodological limitations, which arose in the course of the analysis and which are discussed below.

It seems that the results of the project are largely inconclusive mainly because there is huge variation in the data. This may have resulted from too much research noise generated by a large number of participants and analysed aspects. The all-encompassing nature of the study may have backfired in this case.

Other methodological issues are related to the procedure itself. There was insufficient control of the uniformity of the experimental procedure. Although the same instructions were supposed to be given, the deliverables differed since some participants produced description in only a few sentences. In some countries, the participants were younger (high school students), which may be one of the factors skewing the results. Additionally, the researchers did not control one confounding variable, i.e. the participants' AD experience and training, which may have influenced their descriptions. In the questionnaire part of the study they were not asked about their previous exposure to AD. However, our impression is that not many students were actually familiar with this technique since AD is still a niche in many countries participating in the project. Secondly, the participants were not instructed to provide a description to a blind person, but just to a person who had not seen the film and no reference was made throughout the experiment to the application of the results to AD.

It should also be noted that the study under consideration is a reception-based study where a lot of variation exists not only among the languages involved but also among the subjects within the same language, as reception is very individual and depends on the subject's background, personal experiences or world-view.

The last consideration is more pertinent to the general rationale of the study. The experiment generated casual descriptions whereas $\mathrm{AD}$ offers a well-targeted and specific product. Thus, can we really transfer the results? The answer is not straightforward. Although a casually produced description will definitely differ from a painstakingly forged audio description, we still argue that certain aspects of culture-specific narrative styles (which this study aimed at indicating) might be applicable to AD.

Irrespective of these methodological limitations as well as the findings obtained in the present study, we could assume that common European guidelines could be developed, provided they take into account linguistic and cultural differences as well as preferences of visually challenged audiences in the countries concerned. Nonetheless, more research is 
needed, especially in the form of studies involving eye-tracking methodology, which could yield objective data concerning the perception of visual stimuli. Finally, more reception studies must be done with blind and partially-sighted populations, both at the European and national level, in order to determine their preferences.

\section{Notes}

1. Here is the list of all the languages (including non-European ones) and researchers involved in the project: Afrikaans (J-L. Kruger, N. Wilken, H. Kruger; North-West University, Republic of South Africa), Catalan (A. Matamala, P. Orero, P. Igareda; Universitat Autònoma de Barcelona, Spain), Dutch (A. Remael, H. Rummens, G. Vercauteren; University College Antwerp, Belgium), British English (T. Muller; Roehampton University, United Kingdom), French (A. Serban, T. Muller; Université Paul Valéry/Roehampton University, France/ United Kingdom), German (H. Gerzymisch-Arbogast, A. Gronek, A. Gorius, V. Hildner, J. Fickert and J. Kunold; Universität des Saarlandes, Germany), Greek (S. Sokoli, E. Rapti; Hellenic Open University, Greece), Italian (E. Di Giovanni, S. Giustozzi; Università di Macerata, Italy), Irish English (A. Salway; Dublin City University, Ireland), Polish (A. Chmiel, I. Mazur; Adam Mickiewicz University, Poland), Spanish (P. Igareda; Universitat Autònoma de Barcelona, Spain), Spanish/US English (J. Dávila; University of Texas at Brownsville, USA). Please note that data for some of the above-mentioned languages were first compiled and analysed by Andrew Salway and Alicia Palomo López.

\section{References}

Barlett, F.C. (1967). Remembering. A study in experimental and social sociology. Cambridge: Cambridge University Press.

Chafe, W. (Ed.). (1980). The Pear Stories: Cognitive, cultural and linguistic aspects of narrative production, Norwood, NJ: Ablex.

Du Bois, J.W. (1980). Introduction - The search for a cultural niche: Showing the Pear Film in a Mayan community. In Chafe, W. (Ed.), The Pear Stories: Cognitive, cultural and linguistic aspects of narrative production (1-7). Norwood, NJ: Ablex.

Orero, Pilar. (2008). "Three different receptions of the same film. 'The Pear Stories Project' applied to audio description". European Journal of English Studies, 12(2).

Tannen, D. (1980). A comparative analysis of oral narrative strategies: Athenian Greek and American English. In Chafe, W. (Ed.), The Pear Stories: Cognitive, cultural and linguistic aspects of narrative production (51-87). Norwood, NJ: Ablex. 